\title{
The Utility of Continuous Subcutaneous Insulin Infusion for Management of Mild to Moderate Diabetic Ketoacidosis
}

\author{
Magui Abdel Moneim Shalash, Ali Ahmed Abdel Rahim, Kamel Hemida Rohoma, \\ Marwa Abdel Maaboud Elnabawy
}

Internal Medicine Department, Faculty of Medicine, University of Alexandria, Alexandria, Egypt

Email address:

kamel.hemida@yahoo.com (K. H. Rohoma)

\section{To cite this article:}

Magui Abdel Moneim Shalash, Ali Ahmed Abdel Rahim, Kamel Hemida Rohoma, Marwa Abdel Maaboud Elnabaw. The Utility of Continuous Subcutaneous Insulin Infusion for Management of Mild to Moderate Diabetic Ketoacidosis. American Journal of Internal Medicine. Vol. 4, No. 3, 2016, pp. 43-48. doi: 10.11648/j.ajim.20160403.11

Received: April 13, 2016; Accepted: April 22, 2016; Published: May 11, 2016

\begin{abstract}
Diabetic ketoacidosis (DKA) is the commonest hyperglycemic emergency in people with diabetes. Fluid and insulin, commonly via intravenous route, is the mainstay of treatment; however, other methods of insulin administration have been tried. In this study, we aimed at comparing the efficacy and safety of continuous subcutaneous insulin infusion (CSII) to intravenous (IV) insulin infusion protocol using a short acting insulin analogue, glulisine, in patients with mild to moderate DKA. This is a prospective randomized controlled trial including 30 patients with DKA randomly assigned to receive Glulisine insulin via CSII or IV infusion. Metabolic parameters were observed till resolution of DKA. Primary end point was assessment of the duration till resolution. Secondary end points included total length of hospitalization, amount of insulin used and the number of hypoglycemic events. There were no statistical differences in the mean duration of treatment until correction of DKA being $16.58 \pm 3.68$ hours for CSII group versus $14.60 \pm 3.2$ hours in the IV group, $p=0.136$. There was no mortality and no differences in the length of hospital stay, or the number of hypoglycemic events among treatment groups. However, the total amount of insulin administration until resolution of ketoacidosis was significantly higher, $61.50 \pm 13.89$ units, in CSII group compared to $46.60 \pm 13.53$ units in the IV group, $p=0.009$. We concluded that the use of CSII of glulisine insulin represented a safe and effective alternative to the use of IV glulisine in the management of patients with mild to moderate DKA.
\end{abstract}

Keywords: Diabetes, Ketoacidosis, DKA, Insulin Pump, Continuous Subcutaneous Insulin Infusion

\section{Introduction}

Diabetic ketoacidosis (DKA) is the most common hyperglycemic emergency in people with diabetes and the leading cause of death in children with Type 1 Diabetes Mellitus (T1DM). [1-3] One of the important pillars in treatment of DKA is the administration of regular insulin via intravenous (IV) infusion or by frequent subcutaneous (SC) or intramuscular (IM) injections. [4-6] A number of controlled trials in patients with DKA showed that low-dose insulin therapy is effective no matter by which route it was given. $[7,8]$ Intravenous administration of regular insulin is preferred by most diabetologists because of the delayed onset of action of SC insulin and its prolonged half-life. In their studies, Fisher et al. [9] and Menzel et al. [10] demonstrated that DKA episodes treated with IV regular insulin had experienced a more rapid fall in their plasma glucose and level of blood ketones than those treated with IM or SC insulin. They also showed that more than one third of patients treated with IM or SC insulin did not lower their plasma glucose by $10 \%$ in the first hour of insulin therapy. Yet the cost of treating DKA with IV insulin may be higher, because of the need to admission to an intensive care unit which may not be available in many areas. [11]

New analogues of human insulin with a rapid onset of action, aspart, lispro or Glulisine insulin have become available over the last two decades and may represent an alternative to the use of regular insulin in treatment of DKA. Treatment of mild and moderate DKA with hourly injections of SC lispro insulin was shown to be equally effective as the 
use of the standard IV regular insulin with comparable mean duration of treatment needed to achieve resolution of DKA in both groups. [12] However, treatment with hourly schedule of $\mathrm{SC}$ insulin injections may not be feasible in many hospitals because of nursing staff shortage on regular wards. Therefore, we expanded our investigation on the use of insulin analogue by comparing the use of continuous subcutaneous insulin infusion (CSII) by insulin pump using Glulisine and intravenous Glulisine insulin infusion. CSII has the advantage of consistency of basal insulin delivery with easiness of adjusting its rate and the avoidance of high insulin depot that may precipitate a later hypoglycemia. All these factors advocate its clinical superiority. [13]

\section{Patients and Methods}

\subsection{Recruitment}

This is a prospective, randomized controlled trial that included a total of 30 patients with DKA admitted to Alexandria main university hospital. The diagnosis of DKA was established in the emergency department by a plasma glucose level $>250 \mathrm{mg} / \mathrm{dl}$, a serum bicarbonate level $<15$ $\mathrm{mEq} / \mathrm{L}$, arterial $\mathrm{pH}$ below 7.30 and capillary betahydroxybutyrate $>3.0 \mathrm{mmol} / \mathrm{L}$ (measured with the Precision Xtra glucometer/ketone meter, Abbott Laboratories). [14, 15] Patients with coma, hypovolemic shock, acute myocardial ischemia, end-stage renal or hepatic failure, dementia, pregnancy and sever acidosis were excluded. [16]

\subsection{Randomization}

Out of the 30 subjects admitted to DKA unit of internal medicine department, 15 patients were assigned by simple randomization to receive CSII of glulisine insulin via insulin pump (CSII group); the insulin pump used was "Medtronic minimed 772 paradigm". The other control group included 15 patients who received glulisine insulin via intravenous infusion (IV group).

\subsection{Method}

Both groups started with the same initial dose (0.1 unit $/ \mathrm{kg}$ /hour) of glulisine insulin until blood glucose reached 250 $\mathrm{mg} / \mathrm{dl}$. At that time, insulin dose was reduced to 0.05 units $/ \mathrm{kg} / \mathrm{h}$. [17] Initial Fluid therapy with isotonic saline was given in a rate depending on the clinical state of the patient; isotonic saline was infused at a rate of $10-15 \mathrm{ml} / \mathrm{kg}$ body weight per hour (about $1000 \mathrm{ml} / \mathrm{h}$ in an average-sized person) during the first two hours. [17] Potassium chloride (20 to 30 $\mathrm{mEq} / \mathrm{L}$ ) was added to the replacement fluid once the serum potassium concentration fall below $5.3 \mathrm{mEq} / \mathrm{L}$, with an adequate urine output $(>50 \mathrm{ml} / \mathrm{h})$; aimed to keep serum $\mathrm{K}$ level between 4 and $5 \mathrm{mEq} / \mathrm{L}$. [18] No patients in this study received bicarbonate therapy.

Primary end-point was assessment of the duration of treatment until resolution of ketoacidosis. Additional secondary end points included total length of hospitalization, amount of insulin administration until resolution of ketoacidosis and the number of hypoglycemic events. Hypoglycemia was considered when blood glucose level was $\leq 70 \mathrm{mg} / \mathrm{dl}$. [19]

DKA was considered resolved in the presence of the following: [20]

- Serum bicarbonate $\geq 15 \mathrm{mEq} / \mathrm{L}$

- Arterial $\mathrm{pH}>7.30$

- Capillary $\beta$-hydroxybutyrate $<0.6 \mathrm{mmol} / \mathrm{L}$

When these levels were achieved, the IV infusion of insulin or CSII was discontinued (one hour after the administration of patient's preadmission subcutaneous dose of short and intermediate-acting insulin). [21] Close monitoring was done throughout the treatment duration. Blood glucose was measured by a finger stick every hour. Serum $\mathrm{HCO}_{3}$, arterial $\mathrm{pH}$, and serum $\beta$-hydroxybutyrate were measured on admission (before the initiation of insulin) and at $2,4,8,12,16$, and 24 hours of treatment. Response to medical therapy was evaluated by improvement of metabolic parameters, assessing the time required for resolution of ketoacidosis, the amount of insulin used, total length of hospitalization and the number of hypoglycemic events during therapy.

\subsection{Ethical Approval}

The study was performed according to the guidelines of the Helsinki Declaration [22] and approved by the Ethics Committee of the Alexandria Faculty of Medicine. All participants who freely accepted to participate in the study signed a written informed consent.

\subsection{Statistical Analysis}

Data were analyzed using IBM SPSS software package version 20. [23] Qualitative data were described using number and percent. Quantitative data were described using Range, mean \pm standard deviation and median. Comparison between CSII and IV groups regarding categorical variables was tested using Chi-square test. When more than $20 \%$ of the cells have expected count less than 5, correction for chisquare was conducted using Fisher's Exact test or Monte Carlo correction. The distributions of quantitative variables were tested for normality using Kolmogorov-Smirnov test, Shapiro-Wilk test and D'Agstino test, also Histogram and QQ plot were used for vision test. If it reveals normal data distribution, parametric tests was applied. If the data were abnormally distributed, non-parametric tests were used. For normally distributed data, comparison between CSII and IV were done using independent t-test, while abnormally distributed data was assessed using Mann Whitney test. Significance test results are quoted as two-tailed probabilities. Significance of the obtained results was judged at the $5 \%$ level.

\section{Results}

The study population included 15 patients treated with CSII and 15 patients treated with IV infusion of glulisine 
insulin. The baseline characteristics of both groups showed no significant difference regarding the mean age and duration of diabetes. Both groups consisted of 7 males and 8 females.
The mean random blood glucose, serum bicarbonate, $\mathrm{pH}$ and serum $\beta$-hydroxybutyrate on admission are shown in Table 1.

Table 1. Comparison between the two studied groups according to baseline characteristics.

\begin{tabular}{|c|c|c|c|c|}
\hline & CSII group $(n=15)$ & IV group $(n=15)$ & Test of significance & $\mathbf{p}$ \\
\hline Age (years) & $23.0 \pm 6.83$ & $23.0 \pm 5.88$ & $\mathrm{t}=0.0$ & 1.000 \\
\hline Male/ Female & $7 / 8$ & $7 / 8$ & $\chi 2=0.0$ & 1.000 \\
\hline Duration of diabetes (years) & $9.64 \pm 5.39$ & $9.14 \pm 3.88$ & $\mathrm{Z}=0.277$ & 0.782 \\
\hline blood glucose (mg/dl) & $396.53 \pm 61.92$ & $392.80 \pm 65.46$ & $\mathrm{t}=0.160$ & 0.874 \\
\hline $\mathrm{pH}$ & $7.21 \pm 0.06$ & $7.16 \pm 0.07$ & $\mathrm{t}=0.540$ & 0.561 \\
\hline $\mathrm{HCO} 3(\mathrm{mEq} / \mathrm{L})$ & $12.04 \pm 1.98$ & $11.69 \pm 1.19$ & $\mathrm{t}=0.593$ & 0.558 \\
\hline capillary $\beta$-hydroxybutyrate $(\mathrm{mmol} / \mathrm{L})$ & $5.47 \pm 1.23$ & $5.39 \pm 0.63$ & $Z=0.125$ & 0.901 \\
\hline
\end{tabular}

$\mathrm{p}$ : Statistically significant difference if $\mathrm{p} \leq 0.05$

$\chi 2$ : Chi square test

t: Student t-test

Z: Mann Whitney test

The difference in the decline rate of blood glucose concentration between both groups was statistically significant during the first 8 hours of treatment as it decreased more rapidly in the IV group. It was then insignificant during the rest of follow up as shown in Figure 1. In terms of blood $\mathrm{pH}$, there was no significant difference in the rate of increase between the two groups during the first 8 hours, and then increased significantly more rapidly in the IV group during the next 4 hours. Finally, the rate of $\mathrm{pH}$ increase was not significantly different between both groups during the rest of treatment hours as shown Figure 2. The rate of increase in serum bicarbonate over the treatment period was not significantly different between the two groups. $\beta$ hydroxybutyrate decreased significantly more rapidly in the IV group than the CSII group during the first 8 hours then the difference in the decline rate was insignificant during the rest of treatment hours as shown Figure 3.

The mean duration of treatment until resolution of DKA was not statistically different between the CSII group (16.58 \pm 3.68 hours) versus the IV group (14.60 \pm 30.2 hours), $\mathrm{p}=0.136$. Similarly, the total days of hospitalization were not significantly different between both groups. However, the mean amount of insulin units needed for treatment until resolution of DKA was higher in the CSII group (61.50 \pm 13.89 units $)$ compared to (46.60 \pm 13.53 units) in IV group, $\mathrm{p}=0.009$.

In addition, there was no significant difference in the number of hypoglycemic events during treatment of the two groups; (single event in each of 3 patients in CSII group) versus (single event in each of 2 patients in IV group), $\mathrm{p}=0.605$.

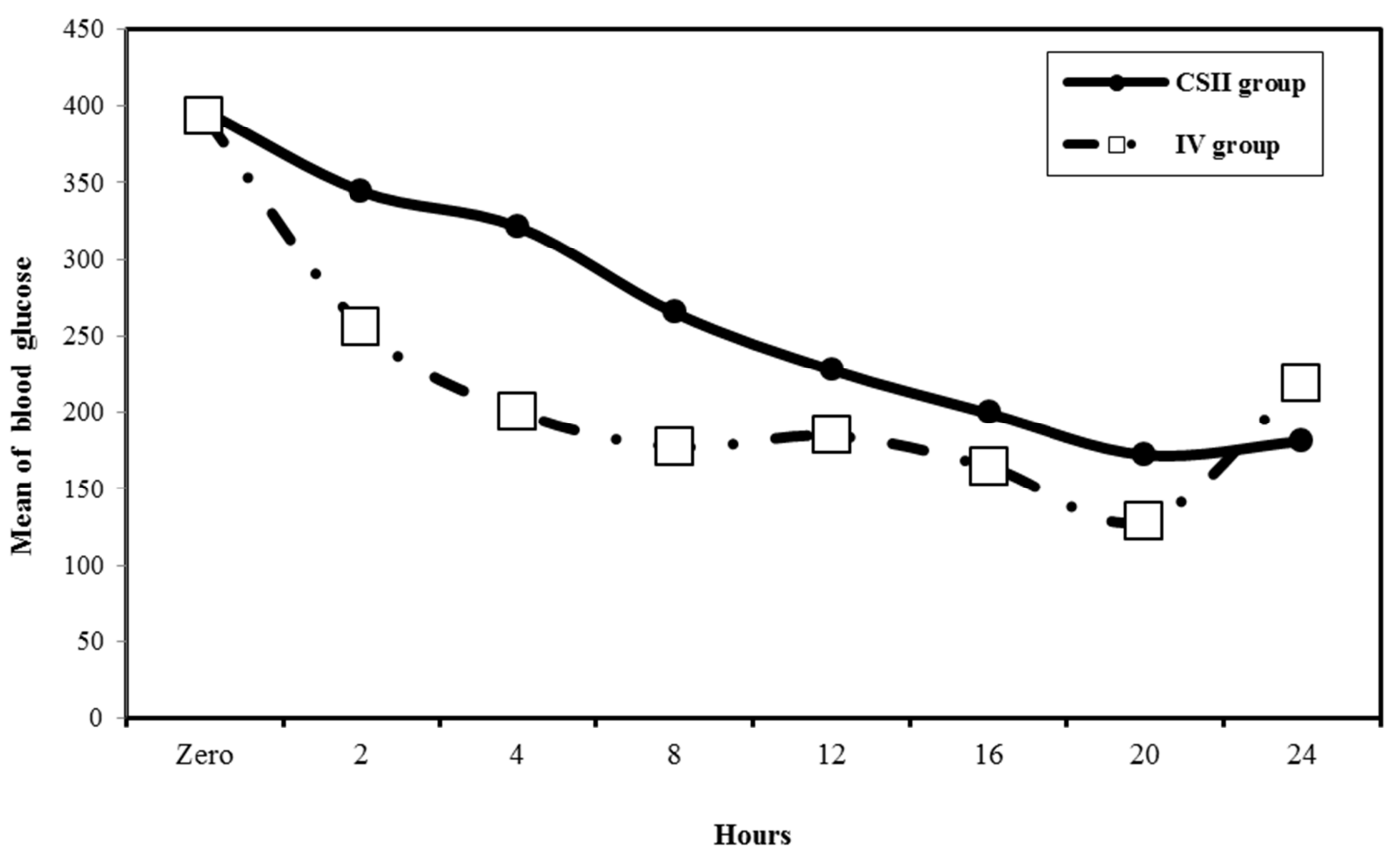

Figure 1. Comparison between the two groups according to the reduction of blood glucose level ( $\mathrm{mg} / \mathrm{dl}$ ). 


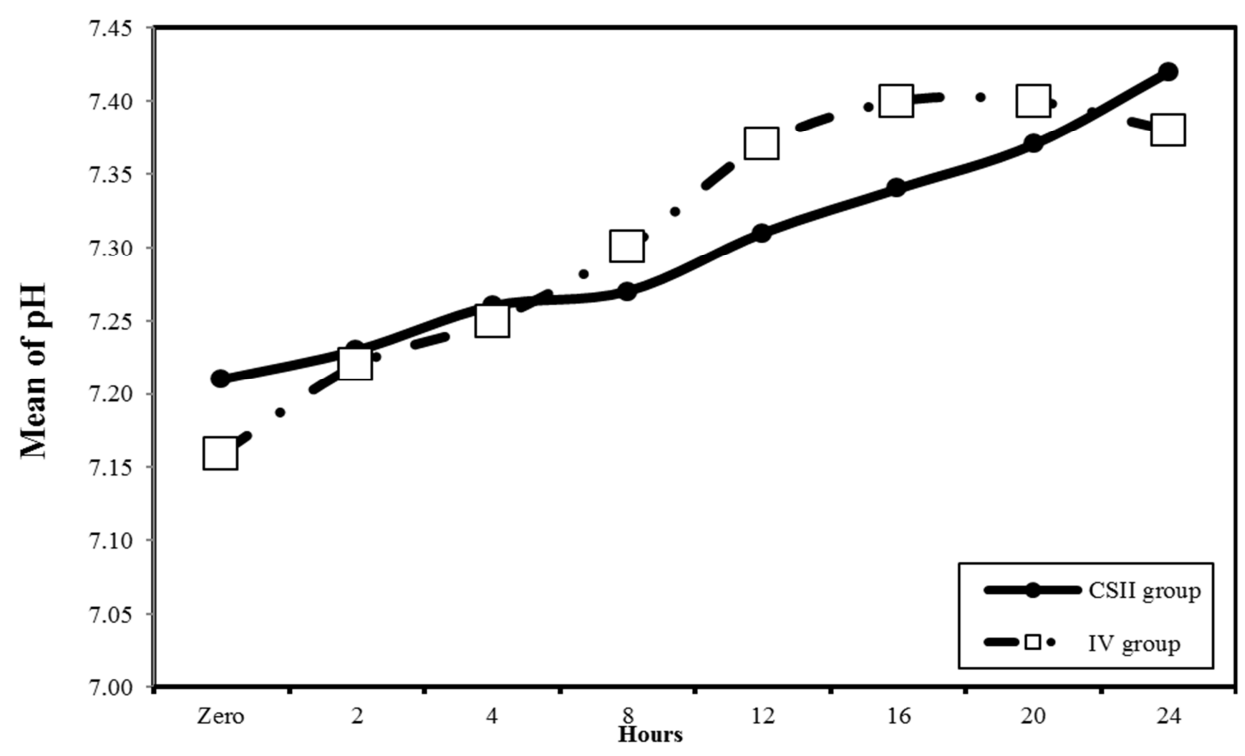

Figure 2. Comparison between the two groups according to the increase in blood pH.

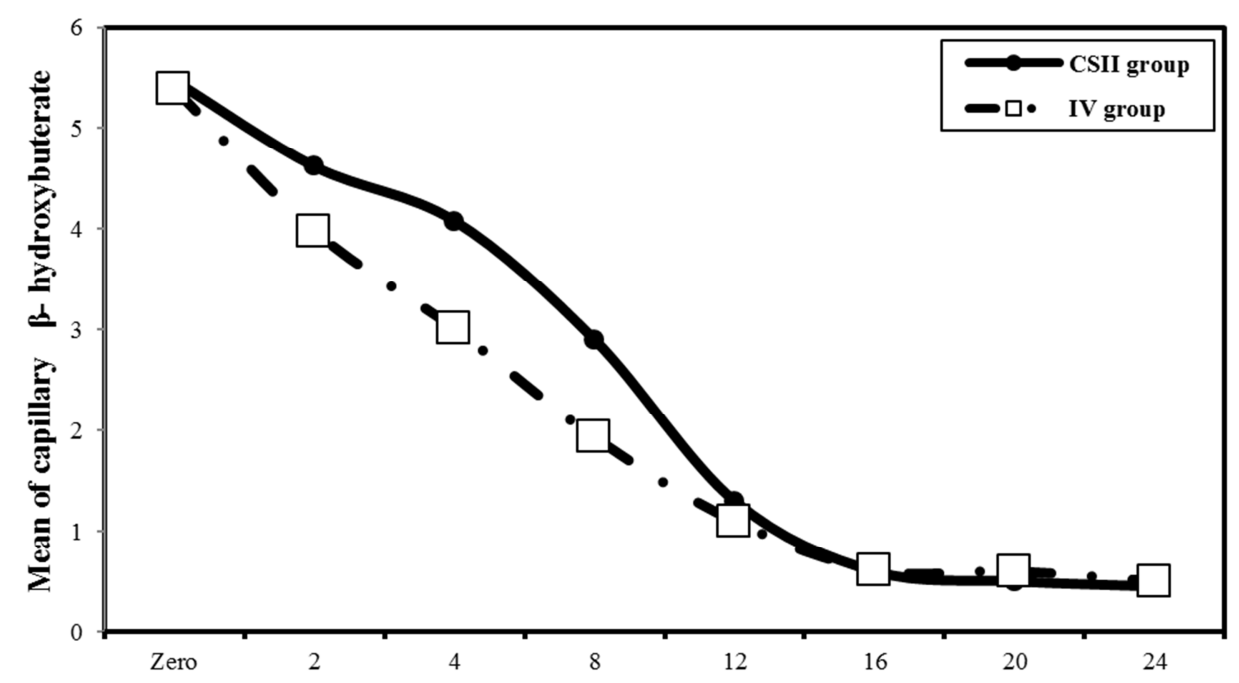

Figure 3. Comparison between the two studied groups according to the reduction of Capillary $\beta$-hydroxybutyrate (mmol/L).

\section{Discussion}

DKA is the most serious hyperglycemic emergency and the leading cause of death in children and adolescents with T1DM. [2] Previous randomized controlled studies in patients with DKA have studied the amount and method of insulin administration required for resolution of ketoacidosis. $[4,5,6]$ However, none of them used the CSII for managing these cases. To our knowledge, this is the first prospective randomized trial to compare the use of continuous subcutaneous insulin infusion of insulin glulisine to intravenous glulisine insulin infusion for management of mild and moderate cases of DKA.

In the past years, Because of fears of insulin resistance, large doses of insulin were thought to be necessary to suppress hepatic ketone body production. [24] However, numerous prospective randomized studies were directed to prove the sufficiency of low-dose regular insulin by intravenous infusion for successful recovery of patients with

\section{DKA. [25, 26]}

Low-dose insulin infusion protocols can decrease plasma glucose concentration at a rate of $50-75 \mathrm{mg} / \mathrm{dl} / \mathrm{h}$. [27] That is why, most medical centers recommend the administration of IV infusion because of the delayed onset of action of SC regular insulin and its prolonged half life. [28] In their prospective randomized study in patients with DKA treated either with IM and SC injections or with continuous IV infusion of regular insulin, Fisher et al. [9] showed that around one third of the patients in the IM and SC groups did not lower their plasma glucose by $10 \%$ in the first hour after insulin injection and that the concentration of ketone bodies was lowered at a significantly faster rate in the IV group.

In our study, there was a significant difference in the decrease of blood glucose level between the two groups during the first 8 hours of treatment with more rapid decrease in the IV treated patients, then no significant difference during the rest of treatment hours. The delay in onset of action of SC insulin is substantiated by the report of Menzel 
and Jutzi, [10] who treated patients with hourly SC injections, but only 4 of 24 patients showed a fall in blood glucose concentration in the first 3 hours of therapy. These differences in response can be explained by delays in reaching a maximal circulating insulin concentration. Maximal insulin peak is achieved within the first hour in patients treated with IV regular insulin, but not until the second or third hour of therapy in those treated with IM or $\mathrm{SC}$ injections.

Reports have showed that treatment of patients with mild and moderate DKA with hourly injections of SC lispro insulin is as effective as treatment with low-dose IV regular insulin. [12] However, this protocol may be difficult to follow in many medical centers because of the intensity of treatment and shortage of nursing staff on general wards.

The mean duration of treatment until resolution of DKA was not statistically different between the CSII group (16.58 \pm 3.68 hours) and IV group (14.60 \pm 30.2 hours), $\mathrm{p}=0.136$. Similarly, the total days of hospitalization were not different between the two groups. However, the mean amount of insulin needed for treatment until resolution of DKA was higher in the CSII group (61.50 \pm 13.89 units) compared to $(46.60 \pm 13.53$ units $)$ in IV group, $(p=0.009)$. There was insignificant difference in the number of hypoglycemic events during therapy ( 3 patients in CSII group and 2 patients in IV group) between the two groups.

Karoli et al. [29] conducted a prospective randomized study that compared the efficacy and safety of subcutaneous insulin analogue every hour with that of standard low dose intravenous infusion of regular insulin in adult patients with DKA. They observed blood glucose decrease rates and control of ketoacidosis between the two groups and concluded that patients with uncomplicated DKA can be managed safely with rapid acting insulin analogue in general medical wards. The rate of decline of blood glucose, time taken for resolution of ketoacidosis, amount of insulin required for improvement of hyperglycemia, and resolution of DKA were similar between the two treatment groups. The duration of hospital stay also was not different between the groups. There was no need to increase or change the dose or route of insulin administration because of delayed or inadequate response in any of the treatment groups. There was no mortality and none of these patients had recurrence of ketoacidosis during their hospital stay.

A Very recent retrospective cohort study, including 76 DKA episodes in children with T1DM, demonstrated that administration of SC regular insulin every 4 hours was a safe and effective alternative for the treatment of DKA with $\mathrm{pH}>$ 7.0. Again, this reinforces the feasibility of using other routes of insulin delivery in management of DKA. [30]

We acknowledge some limitations of our study, including a relatively small number of patients and the fact that patients with coma, hypovolemic shock, acute myocardial ischemia, congestive heart failure, end-stage renal or hepatic failure, and pregnancy were excluded. A large prospective randomized clinical trial of the use of CSII in management of DKA is certainly needed to address these important issues.

\section{Conclusion}

Our results indicate that, until larger studies are done, the use of continuous subcutaneous glulisine insulin infusion represents an effective and safe alternative to the use of intravenous glulisine insulin in the management of patients with uncomplicated mild to moderate DKA. Its effectiveness was shown in terms of normalization of metabolic parameters, the duration of therapy till resolution of DKA and the overall hospital stay that were comparable to the use of intravenous route. There was no increase in hypoglycemic events or mortality with this regimen. However, larger amount of insulin was needed to achieve resolution. We recommend conduction of larger randomized controlled trials on the use of CSII in management of DKA using regular insulin and different rapid acting insulin analogues.

\section{References}

[1] Umpierrez GE, Murphy MB, Kitabchi AE. Diabetic Ketoacidosis and Hyperglycemic Hyperosmolar Syndrome. Diabetes Spectrum 2002; 15: 128-36.

[2] Kitabchi AE, Umpierrez GE, Murphy MB, et al. Hyperglycemic crises in patients with diabetes mellitus. Diabetes Care 2003; 26: 109-17.

[3] Lebovitz HE: Diabetic ketoacidosis. Lancet 2001; 345: $767-72$.

[4] Wallace TM, Matthews DR. Recent advances in the monitoring and management of diabetic ketoacidosis. QJM 2004; 97: 773-80.

[5] Marshall RD, Rand JS, Gunew MN, Menrath VH. Intramuscular glargine with or without concurrent subcutaneous administration for treatment of feline diabetic ketoacidosis. Journal of Veterinary Emergency and Critical Care. 2013; 23 (3): 286-90.

[6] Kitabchi AE. Low-dose insulin therapy in diabetic ketoacidosis. Fact or fiction? Diabetes Metab Rev1989; 5: 337-363.

[7] Sacks HS, Shahshahani M, Kitabchi AE, et al. Similar responsiveness of diabetic ketoacidosis to low-dose insulin by intramuscular injection and albumin-free infusion. Ann Intern Med 1999; 90: 36-42.

[8] Vincent M, Nobécourt E. Treatment of diabetic ketoacidosis with subcutaneous insulin lispro: A review of the current evidence from clinical studies. Diabetes \& metabolism. 2013; 39 (4): 299-305.

[9] Fisher JN, Shahshahani MN, Kitabchi AE. Diabetic ketoacidosis: low-dose insulin therapy by various routes. N Engl J Med 1977; 297: 238-241.

[10] Menzel R, Jutzi E. Blood sugar behavior in recompensation of diabetic coma. Dtsch Gesundheitsw 1970; 25: 727-32.

[11] Freire AX, Umpierrez GE, Afessa B, et al. Predictors of intensive care unit and hospital length of stay in diabetic ketoacidosis. J Crit Care 2002; 17: 207-211. 
[12] Latif K, Umpierrez GE, Stoever J, et al. Subcutaneous lispro insulin in the treatment of diabetic ketoacidosis. Diabetes 2003; 51 (Suppl. 2): 427.

[13] Vasiliki Valla. Continuous Subcutaneous Insulin Infusion (CSII) Pumps. Advances in Experimental Medicine and Biology 2013; 414-419.

[14] Bohan JS. Chemical measurements in ketoacidosis. Arch Intern Med 1999; 159-89.

[15] Voulgari C, Tentolouris N. The performance of a glucoseketone meter in the diagnosis of diabetic ketoacidosis in patients with type 2 diabetes in the emergency room. Diabetes Technol Ther 2010; 12 (7): 529-35.

[16] Lassmann-Vague V, Clavel S, Guerci B, at al. When to treat a diabetic patient using an external insulin pump. Expert consensus. Diabetes Metab 2010; 36: 79-85.

[17] Kitabchi AE, Umpierrez GE, Murphy MB, et al. Management of hyperglycemic crises in patients with diabetes. Diabetes Care 2001; 24: 131-153.

[18] Abramson E, Arky R. Diabetic acidosis with initial hypokalemia. Therapeutic implications. JAMA 1996; 196:401.

[19] Workgroup on Hypoglycemia, American Diabetes Association. Defining and reporting hypoglycemia in diabetes. Diabetes Care 2005; 28: 1245.

[20] Joint British Diabetes Societies Inpatient Care Group. The management of diabetic ketoacidosis in adults, September 2013. http://www.diabetes.org.uk/Documents/About\%20Us/What\% 20we\%20say/Management-of-DKA-241013.pdf. Last accessed 17 May 2015.
[21] Barski L, Kezerle L, Zeller L, et al. New approaches to the use of insulin in patients with diabetic ketoacidosis. Eur J Intern Med 2013; 24: 213-16.

[22] Snežana, Bošnjak. "The declaration of Helsinki: The cornerstone of research ethics". Archive of Oncology 2001; 9: 179-84.

[23] Kirkpatrick LA, Feeney BC. A simple guide to IBM SPSS statistics for version 20.0 Student ed. Belmont, Calif.: Wadsworth, Cengage Learning 2013; 115

[24] Ellemann K, Soerensen JN, Pedersen L, et al. Epidemiology and treatment of diabetic ketoacidosis in a community population. Diabetes Care 1996; 7: 528-532

[25] Genuth SM. Constant intravenous insulin infusion in diabetic ketoacidosis. JAMA 1973; 223: 1348-51.

[26] 26- Edwards GA, Kohaut EC, Wehring B, et al. Effectiveness of low-dose continuous intravenous insulin infusion in diabetic ketoacidosis: a prospective comparative study. J Pediatr 2000; 91: 701-5.

[27] Ebenezer A, Kitabchi AE. Evidence-based management of hyperglycemic emergencies in diabetes mellitus. Diabetes Research And Clinical Practice 2011; 94: 340-51.

[28] Jeannette G, Jeremy G. Hyperglycemic Emergencies in Adults. Canadian Journal of Diabetes 2012; 73: 72-6.

[29] Karoli R, Fatima J, Salman T, et al. Managing diabetic ketoacidosis in non-intensive care unit setting: Role of insulin analogue 2011; 43: 398-401.

[30] Cohen M, Leibovitz N, Shilo S, et al. Subcutaneous regular insulin for the treatment of diabetic ketoacidosis in children. Pediatric Diabetes. 2016 Mar 1. doi: 10.1111/pedi.12380. 